\title{
Exoplanets as Sub-GeV Dark Matter Detectors
}

\author{
Rebecca K. Leane $\oplus^{1,2,{ }^{*}}$ and Juri Smirnov $\circledast^{3,4, \dagger}$ \\ ${ }^{1}$ Center for Theoretical Physics, Massachusetts Institute of Technology, Cambridge, Massachusetts 02139, USA \\ ${ }^{2}$ SLAC National Accelerator Laboratory, Stanford University, Stanford, California 94039, USA \\ ${ }^{3}$ Center for Cosmology and AstroParticle Physics (CCAPP), The Ohio State University, Columbus, Ohio 43210, USA \\ ${ }^{4}$ Department of Physics, The Ohio State University, Columbus, Ohio 43210, USA
}

(Received 8 October 2020; revised 28 November 2020; accepted 15 March 2021; published 22 April 2021)

\begin{abstract}
We present exoplanets as new targets to discover dark matter (DM). Throughout the Milky Way, DM can scatter, become captured, deposit annihilation energy, and increase the heat flow within exoplanets. We estimate upcoming infrared telescope sensitivity to this scenario, finding actionable discovery or exclusion searches. We find that DM with masses above about an $\mathrm{MeV}$ can be probed with exoplanets, with DMproton and DM-electron scattering cross sections down to about $10^{-37} \mathrm{~cm}^{2}$, stronger than existing limits by up to six orders of magnitude. Supporting evidence of a DM origin can be identified through DM-induced exoplanet heating correlated with galactic position, and hence DM density. This provides new motivation to measure the temperature of the billions of brown dwarfs, rogue planets, and gas giants peppered throughout our Galaxy.
\end{abstract}

DOI: 10.1103/PhysRevLett.126.161101

Introduction.-Are we alone in the Universe? This question has driven wide-reaching interest in discovering a planet like our own. Regardless of whether or not we ever find alien life, the scientific advances from finding and understanding other planets will be enormous. From a particle physics perspective, new celestial bodies provide a vast playground to discover new physics.

Astrophysical systems have already been broadly used to probe new physics, including investigating the effects of gravitationally captured dark matter (DM). If there is sufficient gravitational force, deposited DM kinetic energy can noticeably increase the temperature of the system. Regardless of gravitational strength, DM annihilation can also induce heating. This has been investigated in the context of neutron stars and white dwarfs [1-41]. Alternatively, the DM-related heat flow in other moons and planets has been considered, including Earth [42-44], Uranus [45,46], Neptune, and Jupiter [46,47], Mars [44], Earth's Luna [48,49], as well as hot Jupiters [46].

We explore the potential to discover DM using exoplanets-planets outside our solar system. We will use the term "exoplanets" to refer to the broader class of all extrasolar planets (including rogue planets), as well as brown dwarfs, which exist at the planet-star boundary. The general setup of this idea is as follows: particle DM in the galactic halo can scatter with exoplanets, lose energy, and

Published by the American Physical Society under the terms of the Creative Commons Attribution 4.0 International license. Further distribution of this work must maintain attribution to the author(s) and the published article's title, journal citation, and DOI. Funded by SCOAP ${ }^{3}$. become gravitationally captured by the exoplanet. The captured DM accumulates and may annihilate, releasing its mass energy to heat exoplanets. Assuming that the annihilation rate is in equilibrium with the scattering rate (see the Supplemental Material [50]), the annihilation heat measured by upcoming infrared telescopes allows for a new probe of the DM scattering rate.

We will show that this leads to new sensitivities to scattering cross sections between about $10^{-37}-10^{-25} \mathrm{~cm}^{2}$ in the sub-GeV mass range. This range of elastic interactions is expected in models with thermally produced subGeV DM, see, e.g., Refs. [51,52]. This cross section range is bounded by sufficiently weak DM interactions for DM to drift to the core and accumulate, and sufficiently strong DM interactions to produce a detectable DM heat flux. This requires the annihilation rates to be larger than a lower bound provided by the capture and annihilation equilibration condition. For, e.g., $2 \rightarrow 2$ annihilation, we will show that the thermally averaged cross section must be greater than about $10^{-37}-10^{-34} \mathrm{~cm}^{3} / \mathrm{s}$ depending on the target, such that both $s$ - and $p$-wave annihilation can be probed (see Supplemental Material [50] for more details). The lower DM-mass sensitivity is truncated by DM evaporating from the exoplanet (and therefore not annihilating to produce any heat), with sensitivity extending down to about $4 \mathrm{MeV}$ (30 MeV) for brown dwarfs (Jupiters). While higher DM masses can also be probed with exoplanets, we will focus on the $\mathrm{MeV}-\mathrm{GeV}$ mass range, as this features a new cross section range that has not been probed by direct detection or other experiments.

There are many advantages of using exoplanets to search for DM over other celestial bodies. These include the following: 
A rapidly accelerating research program: Until 1992, we did not even know if exoplanets existed. Almost all exoplanets we now know were only discovered in the last decade, with the majority found in the last five years [53]. The exoplanet program is clearly rapidly growing (see the Supplemental Material [50] for details of many new experiments). This provides ample motivation to consider new ways this exploding research area can be used to probe new physics.

Enormous number of expected exoplanets: It is estimated that there is at least one planet per star in our Galaxy, and about one cold planet per star [54]. This means that there should be about 300 billion exoplanets awaiting discovery. While of course these will not all be immediately found, even a small percentage of this number leads to an enormous statistical advantage for understanding potential signals. It also allows ample room for growth with new discoveries and possible surprises in observations. To date, there are 4324 confirmed exoplanets, and an additional 5695 candidates are currently under investigation [53].

Much larger surface area than neutron stars: The other key proposed search using upcoming infrared telescopes on DM-heated astrophysical bodies is with old, cold neutron stars [25]. However, while neutron stars are much more dense, and allow for higher heating rates in part due to enhancements from kinetic heating, exoplanets and brown dwarfs are much larger. A typical neutron star has a radius of about $10 \mathrm{~km}$, while exoplanets of interest have radii of about $50000-200000 \mathrm{~km}$. This means that exoplanet temperatures can be measured much further into the galactic center (GC) and therefore can provide a DM-density dependent heating signal. Exoplanets can also be imaged to much higher significance, and with less exposure time.

Easier to find than neutron stars: The infrared neutron star search requires that a sufficiently cold neutron star candidate at a distance $\lesssim 100 \mathrm{pc}$ from Earth is found [25]. While pulsars have been found at distances of $\sim 100 \mathrm{pc}$ [189], it is possible that a sufficiently cold and sufficiently close-by neutron star may not be found, or cannot be measured with sufficient exposure time. On the other hand, exoplanets outnumber neutron stars in our galaxy by at least about a factor of a thousand [190], and are already known to exist in close enough proximity for DM searches.

Low temperatures: Lastly, exoplanets can be very cold, as they do not undergo nuclear fusion, and can exist very far in large orbits from any host star to which they may be bound. They can even go rogue, floating free from any parent star. As the low temperatures allow for a clearer signal over background for DM heating, exoplanets are advantageous over nuclear-fusing stars. Furthermore, their low core temperatures in part prevent DM evaporation compared to evaporation in these stars, providing new sensitivity to $\mathrm{MeV} \mathrm{DM}$.

In this work, we exploit all these features to identify new searches for DM in exoplanets. We establish two different

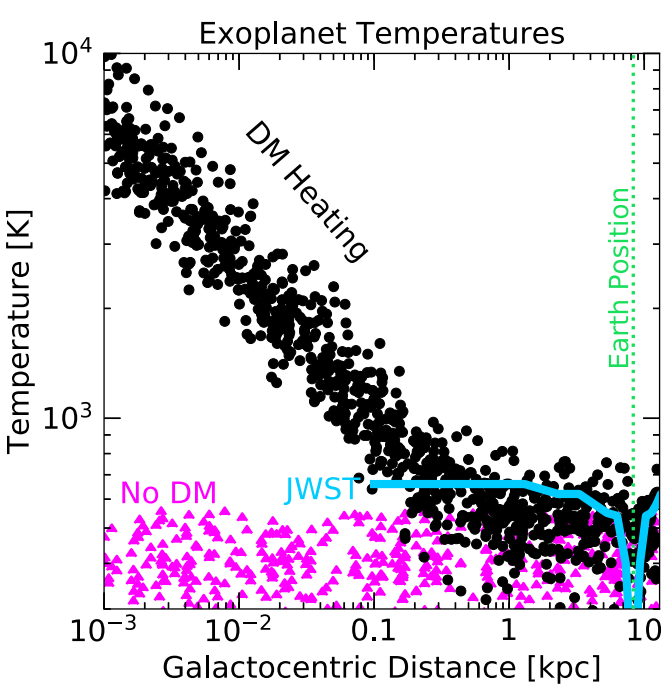

FIG. 1. Mock temperature distribution of old example exoplanets with 20-50 Jupiter masses over Galactocentric distances. Black dots are DM-heated exoplanets, magenta triangles are the same set of planets, without DM heating. JWST is the estimated minimum telescope sensitivity (see text).

searches: one for distant exoplanets and one for local exoplanets. The distant exoplanet searches will require that the exoplanets be rogue (or brown dwarfs), such that their detection is not obscured.

Figure 1 demonstrates these searches and shows an example distribution of exoplanets with masses of about 20-50 Jupiters with and without DM heating. Distant exoplanets can be used to map the Galactic DM density, given sufficient telescope sensitivity. This is seen by the uptick of many hot exoplanets, scaling with the DM density. As well as searching for DM signals, local exoplanets can be used to test the hypothesis that DM contributes to internal heat of the gas giants in our own solar system, which are not well understood $[46,47]$. DMheated exoplanets can be potentially measured when the infrared telescope James Webb Space Telescope (JWST) comes online. Both our suggested searches target new DM parameter space, probing the DM-proton and DM-electron scattering cross sections to unprecedented sensitivities.

Dark heat flow in exoplanets. - The total heat flow of the exoplanet $\Gamma_{\text {heat }}^{\text {tot }}$ can be determined by combining potential heat power sources, including internal heat $\Gamma_{\text {heat }}^{\text {int }}$, external heat $\Gamma_{\text {heat }}^{\text {ext }}$, and DM heat $\Gamma_{\text {heat }}^{\text {DM }}$ :

$$
\Gamma_{\text {heat }}^{\text {tot }}=\Gamma_{\text {heat }}^{\text {ext }}+\Gamma_{\text {heat }}^{\text {int }}+\Gamma_{\text {heat }}^{\mathrm{DM}}=4 \pi R^{2} \sigma_{\mathrm{SB}} T^{4} \epsilon,
$$

where $R$ is the exoplanet radius, $T$ is the exoplanet temperature, $\sigma_{\mathrm{SB}}$ is the Stefan-Boltzmann constant, and $\epsilon$ is the emissivity (which is a measure of planetary heat radiation efficiency, ranging from 0 to 1 ). External heating is negligible for wide-orbit or free-floating planets. 
We compute the internal heat flow for our range of benchmark brown dwarfs and Jupiters without DM. As the minimum temperature for heavy brown dwarfs (with $55 M_{\text {jup }}$ ) and benchmark Jupiters (with $M_{\text {jup }}$ ) after about $10 \mathrm{Gyr}$ is about 750 and $80 \mathrm{~K}$, respectively, we can determine the internal heat flow required to produce these temperatures,

$$
\Gamma_{\text {heat }}^{\text {int }}=4 \pi R^{2} \sigma_{\mathrm{SB}} T^{4} \epsilon,
$$

which corresponds to about $1.1 \times 10^{9} \mathrm{TW}$ and $1.4 \times$ $10^{5}$ TW for benchmark brown dwarfs and Jupiters, respectively. This serves as our non-DM baseline for comparing with a potential DM signal.

The additional DM heating source occurs if DM scatters on exoplanet particles, becomes captured, and annihilates. This produces heat that can be absorbed by the exoplanet. We assume that the DM scattering and annihilation processes are in equilibrium (see the Supplemental Material [50]). The DM heat flow depends on how many external DM particles are captured from the incoming DM flux reservoir. The maximal, i.e., geometric capture rate of DM is given by [191]

$$
C_{\max }=\pi R^{2} n_{\chi}(r) v_{0}\left(1+\frac{3}{2} \frac{v_{\mathrm{esc}}^{2}}{v_{d}(r)^{2}}\right) \xi\left[v_{p}, v_{d}(r)\right],
$$

where $n_{\chi}(r)$ is the DM number density at distance $r$ from the GC, the average speed in the DM rest frame $v_{0}$ is related to the velocity dispersion $v_{d}(r)$ as $v_{0}=\sqrt{8 /(3 \pi)} v_{d}(r)$ at distance $r$ from the GC, and $R$ is the exoplanet radius. The factor $1+3 v_{\text {esc }}^{2} / 2 v_{d}^{2}$ is the result of gravitational focusing, with $v_{\text {esc }}^{2}=2 G_{N} M / R$ being the escape velocity, $M$ the exoplanet mass, and $G_{N}$ the gravitational constant. The motion of the planet with velocity $v_{p}$ with respect to the DM halo is taken into account by $\xi\left[v_{p}, v_{d}(r)\right]$. In the scenarios we are interested in, the DM velocity, the planetary velocity and the escape velocities are of similar order and the function $\xi\left[v_{d}(r), v_{p}\right] \sim 1$. The circular velocities $v_{c}(r)$ in the galaxy are related to the DM velocity dispersion by $v_{d}(r)=\sqrt{3 / 2} v_{c}(r)$. We extract the circular velocities at different radii in the Milky Way by combining the data for the gas, bulge, and disk components, as well as the analytic expressions for DM contributions to the total velocity from Ref. [192]. For the DM density, we consider an Navarro-Frenk-White (NFW) profile [55], a generalized Navarro-Frenk-White (gNFW) profile, and a Burkert profile [193], with the local DM density $0.42 \mathrm{GeV} / \mathrm{cm}^{3}$ [194], see the Supplemental Material [50] for more details.

The heat power produced by DM is given by the product of the DM mass $m_{\chi}$, the fraction of the captured DM particles that have passed through the object $f$, and the maximal capture rate, such that

$$
\Gamma_{\text {heat }}^{\text {DM }}=m_{\chi} f C_{\max } \cdot
$$

Using $n_{\chi}(r)=\rho_{\chi}(r) / m_{\chi}, \xi\left[v_{d}(r), v_{p}\right] \sim 1$, and combining with Eq. (3), the DM heat power is

$$
\Gamma_{\text {heat }}^{\mathrm{DM}}=f \pi R^{2} \rho_{\chi}(r) v_{0}\left(1+\frac{3}{2} \frac{v_{\mathrm{esc}}^{2}}{v_{d}(r)^{2}}\right) .
$$

Searches and infrared telescope sensitivity.-Exoplanets may first be identified by, e.g., Doppler spectroscopy or gravitational lensing. Once their location is found, infrared telescopes such as JWST may be able to measure their temperature. The general sensitivity of JWST to exoplanet heating can be found by considering the spectral flux density,

$$
f_{\nu}=\pi B(\nu, T) \times \frac{4 \pi R^{2}}{4 \pi d^{2}},
$$

where $d$ is the distance from the telescope to the exoplanet, $R$ is the radius of the exoplanet, and

$$
B(\nu, T)=\frac{2 \nu^{3} \epsilon}{\exp \left(\frac{2 \pi \nu}{k_{b} T}\right)-1},
$$

where $\nu$ is the wavelength, $T$ is the temperature, $k_{b}$ is the Boltzmann constant, and $\epsilon$ is the atmospheric emissivity. We use $\epsilon=1$ which provides the usual blackbody spectral flux density, and is the most conservative case. Deviations from a blackbody occur for $\epsilon<1$; see the Supplemental Material [50] for emissivity impact on telescope sensitivity.

Figure 2 shows the expected exoplanet temperature as a function of Galactocentric distance, for DM-heating arising due to several different DM profiles as labeled. We distinguish between Jovian exoplanets with masses between $1-14 M_{\text {jup }}$ and brown dwarfs with masses in the range of $14-55 M_{\text {jup }}$. All exoplanets shown have a radius of $R_{\text {jup }}$, as they are expected to converge to this radius after $10 \mathrm{~Gy}$. The shaded region for a given DM profile represents the range of heating possibilities for the indicated mass range, with the heaviest (lightest) exoplanets lying at the upper (lower) boundary. The shape of the curves over galactic distances is due to an interplay of the DM density and velocity profiles, and the effective capture radius of the exoplanet.

We show in Fig. 2 the optimal JWST sensitivity, which is found using Eq. (6) with the benchmark dwarf/Jupiter radius of $R_{\text {jup }}$. As different JWST instrument filters are optimized for different flux densities/temperatures, we use several different filters while scanning over the minimal temperature measurable, to obtain the optimal sensitivity. This is calculated using the near-infrared imager and slitless spectrograph in imaging mode for temperatures above about $500 \mathrm{~K}$, and the mid-infrared instrument in imaging or medium-resolution spectroscopy mode for temperatures from about $100-500 \mathrm{~K}$. The dashed line is for JWST to obtain about $10^{5}$ seconds of exposure to achieve a 

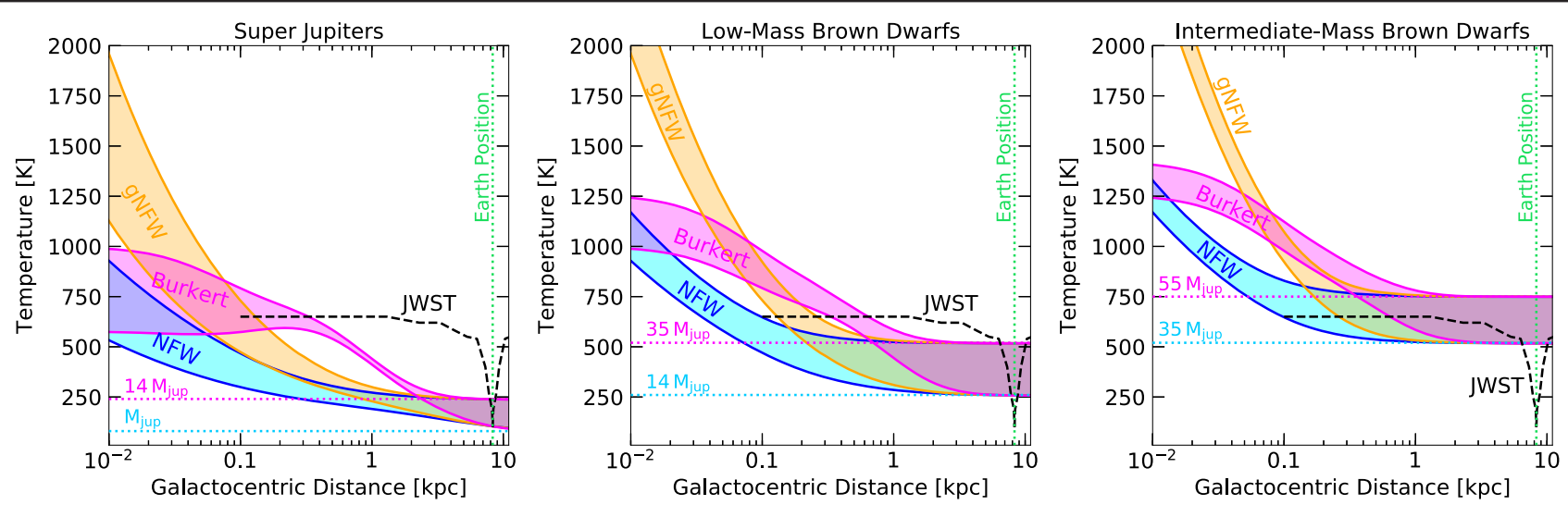

FIG. 2. Exoplanet temperatures over Galactocentric distances, with variations due to DM for labeled density profiles. Each panel represents our classification of different exoplanet types: super Jupiters $\left(M_{\text {jup }}-14 M_{\text {jup }}\right)$, low-mass brown dwarfs $\left(14-35 M_{\text {jup }}\right)$, and intermediate-mass brown dwarfs $\left(35-55 M_{\text {jup }}\right)$. Any exoplanet within the indicated mass range will have temperatures between these lines in the shaded region. The dotted lines show the range of minimum temperatures for a 10 Giga-year-old exoplanet without DM. Black dashed line is the optimal minimum JWST sensitivity (temperatures above this line can be detected), see text.

signal-to-noise ratio (SNR) of 2. 10 SNR can be achieved at about $10^{6}$ seconds of exposure at most of the temperatures shown. Note however that these exposure times are for the minimum temperatures on the dashed line; higher temperatures generally require less exposure time. Significantly less time is required to achieve $10 \mathrm{SNR}$ in the local region; see Supplemental Material for more detailed JWST sensitivity estimates, including estimates of dust extinction and stellar crowding, which conservatively limit the expected JWST sensitivity to distances larger than $0.1 \mathrm{kpc}$ from the GC.

The dotted lines in Fig. 2 show exoplanet temperatures without DM heating, for masses labeled, where intermediate masses would sit between these dotted lines. It is clear that different types of exoplanets are most useful as a DM heating targets in different regimes. The lower mass Jupiters are ideal for local searches, as DM heating can outperform their internal heat at the local position. For higher mass brown dwarfs, their internal heat is too high to reveal a DM heating signal at the local position. However, their larger masses are advantageous towards the GC due to gravitational focusing.

We therefore propose two search strategies: one for DM in local Jupiters, and another for all exoplanets towards the DM dense GC. As shown in Fig. 2, towards the GC exoplanets are increasingly heated by DM. This means that exoplanets can potentially be used to trace the DM density in our Galaxy. Given a large statistical sample, DM overdensities could also be revealed by too many hot exoplanets in a given region, which is additional motivation for the distant search. Note that at large distances the exoplanet must be a rogue planet or brown dwarf, since a parent star would obscure its resolution.

Dark matter parameter space.-We now consider the implications of DM-heated exoplanets for particle DM models. Limits from planetary heat flow often probe strongly interacting DM $[42,44]$. Exoplanets are instead ideal laboratories to study broader classes of sub-GeV DM models.

To relate the DM heat flow with scattering cross sections, we need to find the range of parameters for which a fraction $f$ of the DM particles passing through the planet is gravitationally captured. Normalizing to the maximal DM capture rate [defined in Eq. (3)], we obtain the captured DM fraction

$$
f=\frac{C_{\text {cap }}}{C_{\max }}=\sum_{N=1}^{N_{\max }} f_{N},
$$

with the capture fraction for a given number of scatterings being

$$
f_{N}=p(N, \tau)\left[1-\kappa \exp \left(-\frac{3\left(v_{N}^{2}-v_{\mathrm{esc}}^{2}\right)}{2 v_{d}^{2}}\right)\right]
$$

with

$$
\kappa=\left(1+\frac{3}{2} \frac{v_{N}^{2}}{v_{d}^{2}}\right)\left(1+\frac{3}{2} \frac{v_{\mathrm{esc}}^{2}}{v_{d}^{2}}\right)^{-1} .
$$

Here $v_{d}$ is the velocity dispersion, $v_{N}=v_{\text {esc }}(1-\langle z\rangle \beta)^{-N / 2}$ where the average scattering angle is $\langle z\rangle=1 / 2$ [195], $\beta=4 m_{\chi} m_{A} /\left(m_{\chi}+m_{A}\right)^{2}$, and $m_{A}$ is the mass of the target particle. The probability that the DM particle scatters $N$ times is

$$
p(N, \tau)=\frac{2}{\tau^{2}}\left(N_{s}+1-\frac{\Gamma\left(N_{s}+2, \tau\right)}{N_{s} !}\right),
$$

where $\Gamma(a, b)$ is the incomplete gamma function. This scattering probability is a function of the optical depth, 
$\tau=\frac{3}{2} \sigma / \sigma_{\text {sat }}$ where $\sigma_{\text {sat }}=\pi R^{2} / N_{\mathrm{SM}}$ is the saturation cross section, $R$ the planetary radius, $N_{\mathrm{SM}}$ is the target particle number, and $\sigma$ is the DM-target cross section. To set sensitivity limits on DM scattering in Jupiters and brown dwarfs, we assume spheres of hydrogen with constant density. As gas giants are expected to be dominated by hydrogen, we expect a hydrogen sphere to be approximately representative. For example, Jupiter's composition is about $84 \%$ hydrogen and $16 \%$ helium [196].

Figure 3 shows our sensitivity estimates for Jupiter-like planets and brown dwarfs to the DM parameter space for spin-dependent DM-proton scattering (see Sec. V of the Supplemental Material [50] for spin-independent, additional velocity dependent, and electron scattering results). We show a "min" cross section for the object as labeled, which corresponds to effectively all the DM being captured, and planets being maximally heated, producing the most striking signal. We also show a "max" cross section, which corresponds to the smallest DM capture fraction (about 10\%) that can be probed in the near future with JWST. This is likely the maximum cross section reach, as the temperatures corresponding to this lower scattering rate are approaching either the JWST minimum temperature detection threshold, or the expected background temperature, in most of the parameter space. The sensitivity curves become flat for brown dwarfs, as their larger escape velocity leads to the opacity limit being reached for some of the parameter space. Thus, increasing the DM capture cross section further in that region does not change the capture fraction.

These sensitivities do not depend on the DM density profile or value; sensitivity to the cross sections shown only requires that the DM heating is successfully detected. For

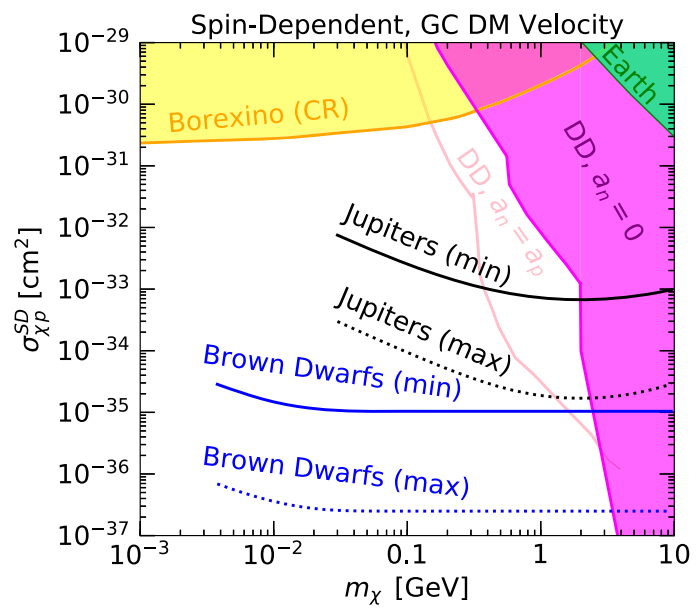

FIG. 3. Spin-dependent DM-nucleon scattering cross section sensitivity estimates for Jupiters and brown dwarfs, assuming a GC DM velocity. The solid (min) lines show cross sections assuming effectively that all DM is captured, and the dotted $(\max )$ lines show the maximum expected reach. Complementary constraints are also shown, see text. more discussion of detection/search prospects, see the Supplemental Material [50]. We show the earth heat flow bounds from Ref. [44] for comparison, and direct detection (DD) bounds $[56,57,197,198]$. "DD, $a_{n}=0$ " corresponds to proton-only DD limits, while " $\mathrm{DD}, a_{n}=a_{p}$ " corresponds to equal proton/neutron coupling DD limits. Borexino shows limits from boosted DM from cosmic rays interactions [58-61]. Note that these limits have different assumptions; the direct detection limits do not require any minimum annihilation cross section.

Conclusions.-The exoplanet program is rapidly accelerating. Amongst the billions of new worlds in our Galaxy, many are waiting to reveal their surprises. Unexpected discoveries are inevitable, and numerous new telescopes with cutting-edge technology are ready to make them. For the first time, we have pointed out the broad applicability of exoplanets to be used as DM detectors, with actionable discovery or exclusion searches using new infrared telescopes. We target old, cold, Jupiter-like planets and brown dwarfs, which are advantageous due to their large sizes, densities, and low core temperatures.

Our first suggested search is for overheated local exoplanets. There are hundreds of known Jupiters in our local neighborhood, and Gaia is expected to identify tens of thousands of potential candidates in the next few years [93], providing a sample with great statistical power.

Our second suggested search is for overheated exoplanets, correlated with DM density, rising sharply in temperature towards the GC. The presence of DM overdensities or substructure may also be confirmed with exoplanets, with a pocket of even hotter DM-heated exoplanets. We conclude that, at an estimate, JWST may have sensitivity to exoplanet temperatures above about $650 \mathrm{~K}$, for exoplanets all the way into about $0.1 \mathrm{kpc}$ of the inner Galaxy (for more local searches, the minimum temperature sensitivity is lower).

We calculated the DM parameter space sensitivity to brown dwarfs and Jupiters that may have their temperature measured in the near future. We determined that DM with masses above about an $\mathrm{MeV}$ can be probed with exoplanets, with DM-proton scattering cross sections down to about $10^{-37} \mathrm{~cm}^{2}$, stronger than existing limits by up to six orders of magnitude. We pointed out that this DM mass sensitivity is lighter than many other celestial body searches for DM heat flow. This is because brown dwarfs and Jupiters have large integrated column densities and low core temperatures, and so it is more difficult for light DM to evaporate in these systems.

We thank John Beacom, Joe Bramante, Chris Cappiello, Rouven Essig, Katie Freese, Savannah Jacklin, Shirley Li, Bruce Macintosh, Nirmal Raj, Anupam Ray, Pat Scott, Sara Seager, Aaron Vincent, and Ji Wang for helpful comments and discussions. R. K. L. was supported by the Office of High Energy Physics of the U.S. Department of Energy under Grants No. DE-SC00012567 and No. DESC0013999, as well as the NASA Fermi Guest Investigator 
Program under Grant No. 80NSSC19K1515, and later at SLAC under Contract No. DE-AC02-76SF00515. J. S. is largely supported by a Feodor Lynen Fellowship from the Alexander von Humboldt foundation.

*rleane@slac.stanford.edu

†smirnov.9@osu.edu

[1] I. Goldman and S. Nussinov, Weakly interacting massive particles and neutron stars, Phys. Rev. D 40, 3221 (1989).

[2] A. Gould, B. T. Draine, R. W. Romani, and S. Nussinov, Neutron stars: Graveyard of charged dark matter, Phys. Lett. B 238, 337 (1990).

[3] C. Kouvaris, WIMP annihilation and cooling of neutron stars, Phys. Rev. D 77, 023006 (2008).

[4] G. Bertone and M. Fairbairn, Compact stars as dark matter probes, Phys. Rev. D 77, 043515 (2008).

[5] A. de Lavallaz and M. Fairbairn, Neutron stars as dark matter probes, Phys. Rev. D 81, 123521 (2010).

[6] C. Kouvaris and P. Tinyakov, Can neutron stars constrain dark matter?, Phys. Rev. D 82, 063531 (2010).

[7] S. D. McDermott, H.-B. Yu, and K. M. Zurek, Constraints on scalar asymmetric dark matter from black hole formation in neutron stars, Phys. Rev. D 85, 023519 (2012).

[8] C. Kouvaris and P. Tinyakov, Excluding Light Asymmetric Bosonic Dark Matter, Phys. Rev. Lett. 107, 091301 (2011).

[9] T. Guver, A. E. Erkoca, M. Hall Reno, and I. Sarcevic, On the capture of dark matter by neutron stars, J. Cosmol. Astropart. Phys. 05 (2014) 013.

[10] J. Bramante, K. Fukushima, and J. Kumar, Constraints on bosonic dark matter from observation of old neutron stars, Phys. Rev. D 87, 055012 (2013).

[11] N. F. Bell, A. Melatos, and K. Petraki, Realistic neutron star constraints on bosonic asymmetric dark matter, Phys. Rev. D 87, 123507 (2013).

[12] J. Bramante, K. Fukushima, J. Kumar, and E. Stopnitzky, Bounds on self-interacting fermion dark matter from observations of old neutron stars, Phys. Rev. D 89, 015010 (2014).

[13] B. Bertoni, A. E. Nelson, and S. Reddy, Dark matter thermalization in neutron stars, Phys. Rev. D 88, 123505 (2013).

[14] C. Kouvaris and P. Tinyakov, Constraining Asymmetric Dark Matter through observations of compact stars, Phys. Rev. D 83, 083512 (2011).

[15] M. McCullough and M. Fairbairn, Capture of inelastic dark matter in white dwarves, Phys. Rev. D 81, 083520 (2010).

[16] M. Angeles Perez-Garcia and J. Silk, Constraining decaying dark matter with neutron stars, Phys. Lett. B 744, 13 (2015).

[17] J. Bramante, Dark Matter Ignition of Type Ia Supernovae, Phys. Rev. Lett. 115, 141301 (2015).

[18] P. W. Graham, S. Rajendran, and J. Varela, Dark matter triggers of supernovae, Phys. Rev. D 92, 063007 (2015).

[19] M. Cermeno, M. A. Perez-Garcia, and J. Silk, Light dark matter scattering in outer neutron star crusts, Phys. Rev. D 94, 063001 (2016).
[20] P. W. Graham, R. Janish, V. Narayan, S. Rajendran, and P. Riggins, White dwarfs as dark matter detectors, Phys. Rev. D 98, 115027 (2018).

[21] J. F. Acevedo and J. Bramante, Supernovae sparked by dark matter in white dwarfs, Phys. Rev. D 100, 043020 (2019).

[22] R. Janish, V. Narayan, and P. Riggins, Type Ia supernovae from dark matter core collapse, Phys. Rev. D 100, 035008 (2019).

[23] R. Krall and M. Reece, Last electroweak WIMP standing: Pseudo-dirac Higgsino status and compact stars as future probes, Chin. Phys. C 42, 043105 (2018).

[24] D. McKeen, A. E. Nelson, S. Reddy, and D. Zhou, Neutron Stars Exclude Light Dark Baryons, Phys. Rev. Lett. 121, 061802 (2018).

[25] M. Baryakhtar, J. Bramante, S. W. Li, T. Linden, and N. Raj, Dark Kinetic Heating of Neutron Stars and An Infrared Window On WIMPs, SIMPs, and Pure Higgsinos, Phys. Rev. Lett. 119, 131801 (2017).

[26] N. Raj, P. Tanedo, and H.-B. Yu, Neutron stars at the dark matter direct detection frontier, Phys. Rev. D 97, 043006 (2018).

[27] N. F. Bell, G. Busoni, and S. Robles, Heating up neutron stars with inelastic dark matter, J. Cosmol. Astropart. Phys. 09 (2018) 018.

[28] R. Garani, Y. Genolini, and T. Hambye, New analysis of neutron star constraints on asymmetric dark matter, J. Cosmol. Astropart. Phys. 05 (2019) 035.

[29] C.-S. Chen and Y.-H. Lin, Reheating neutron stars with the annihilation of self-interacting dark matter, J. High Energy Phys. 08 (2018) 069.

[30] B. Dasgupta, A. Gupta, and A. Ray, Dark matter capture in celestial objects: Improved treatment of multiple scattering and updated constraints from white dwarfs, J. Cosmol. Astropart. Phys. 08 (2019) 018.

[31] K. Hamaguchi, N. Nagata, and K. Yanagi, Dark matter heating vs. rotochemical heating in old neutron stars, Phys. Lett. B 795, 484 (2019).

[32] D. A. Camargo, F. S. Queiroz, and R. Sturani, Detecting dark matter with neutron star spectroscopy, J. Cosmol. Astropart. Phys. 09 (2019) 051.

[33] N. F. Bell, G. Busoni, and S. Robles, Capture of leptophilic dark matter in neutron stars, J. Cosmol. Astropart. Phys. 06 (2019) 054.

[34] R. Garani and J. Heeck, Dark matter interactions with muons in neutron stars, Phys. Rev. D 100, 035039 (2019).

[35] J. F. Acevedo, J. Bramante, R. K. Leane, and N. Raj, Warming nuclear pasta with dark matter: Kinetic and annihilation heating of neutron star crusts, J. Cosmol. Astropart. Phys. 03 (2020) 038.

[36] A. Joglekar, N. Raj, P. Tanedo, and H.-B. Yu, Relativistic capture of dark matter by electrons in neutron stars, Phys. Lett. B 809, 135767 (2020).

[37] A. Joglekar, N. Raj, P. Tanedo, and H.-B. Yu, Kinetic heating from contact interactions with relativistic targets: Electrons capture dark matter in neutron stars, Phys. Rev. D 102, 123002 (2020).

[38] N. F. Bell, G. Busoni, S. Robles, and M. Virgato, Improved treatment of dark matter capture in neutron stars, J. Cosmol. Astropart. Phys. 09 (2020) 028. 
[39] B. Dasgupta, A. Gupta, and A. Ray, Dark matter capture in celestial objects: Light mediators, self-interactions, and complementarity with direct detection, J. Cosmol. Astropart. Phys. 10 (2020) 023.

[40] R. Garani, A. Gupta, and N. Raj, Observing the thermalization of dark matter in neutron stars, Phys. Rev. D 103, 043019 (2021).

[41] R. K. Leane, T. Linden, P. Mukhopadhyay, and N. Toro, Celestial-body focused dark matter annihilation throughout the galaxy, arXiv:2101.12213.

[42] G. D. Mack, J. F. Beacom, and G. Bertone, Towards closing the window on strongly interacting dark matter: Far-reaching constraints from Earth's heat flow, Phys. Rev. D 76, 043523 (2007).

[43] B. Chauhan and S. Mohanty, Constraints on leptophilic light dark matter from internal heat flux of Earth, Phys. Rev. D 94, 035024 (2016).

[44] J. Bramante, A. Buchanan, A. Goodman, and E. Lodhi, Terrestrial and martian heat flow limits on dark matter, Phys. Rev. D 101, 043001 (2020).

[45] S. Mitra, Uranus' anomalously low excess heat constrains strongly interacting dark matter, Phys. Rev. D 70, 103517 (2004).

[46] S. L. Adler, Planet-bound dark matter and the internal heat of Uranus, Neptune, and hot-Jupiter exoplanets, Phys. Lett. B 671, 203 (2009).

[47] M. Kawasaki, H. Murayama, and T. Yanagida, Can the strongly interacting dark matter be a heating source of Jupiter?, Prog. Theor. Phys. 87, 685 (1992).

[48] R. Garani and P. Tinyakov, Constraints on dark matter from the moon, Phys. Lett. B 804, 135403 (2020).

[49] M. H. Chan and C. M. Lee, Constraining the spinindependent elastic scattering cross section of dark matter using the Moon as a detection target and the background neutrino data, Phys. Rev. D 102, 023024 (2020).

[50] See Supplemental Material at http://link.aps.org/ supplemental/10.1103/PhysRevLett.126.161101 for further details on exoplanets, particle DM models, additional search details and cross section sensitivities, which includes Refs. [44,51-188].

[51] Y. Hochberg, E. Kuflik, T. Volansky, and J. G. Wacker, Mechanism for Thermal Relic Dark Matter of Strongly Interacting Massive Particles, Phys. Rev. Lett. 113, 171301 (2014).

[52] J. Smirnov and J. F. Beacom, Co-SIMP Miracle, Phys. Rev. Lett. 125, 131301 (2020).

[53] Nasa exoplanet catalog, (2020).

[54] A. Cassan, D. Kubas, J.-P. Beaulieu, M. Dominik, K. Horne, J. Greenhill, J. Wambsganss, J. Menzies, A. Williams, U. G. Jørgensen et al., One or more bound planets per Milky Way star from microlensing observations, Nature (London) 481, 167 (2012).

[55] J. F. Navarro, C. S. Frenk, and S. D. M. White, The structure of cold dark matter halos, Astrophys. J. 462, 563 (1996).

[56] P. Di Gangi (XENON Collaboration), Results of the 1 tonne D7 year WIMP search with XENON1T, Nuovo Cimento C 42, 76 (2019).

[57] E. Aprile et al. (XENON Collaboration), Constraining the Spin-Dependent WIMP-Nucleon Cross Sections with XENON1T, Phys. Rev. Lett. 122, 141301 (2019).
[58] C. V. Cappiello, K. C. Y. Ng, and J. F. Beacom, Reverse direct detection: Cosmic Ray scattering with light dark matter, Phys. Rev. D 99, 063004 (2019).

[59] T. Bringmann and M. Pospelov, Novel Direct Detection Constraints on Light Dark Matter, Phys. Rev. Lett. 122, 171801 (2019).

[60] Y. Ema, F. Sala, and R. Sato, Light Dark Matter at Neutrino Experiments, Phys. Rev. Lett. 122, 181802 (2019).

[61] C. Cappiello and J. F. Beacom, Strong new limits on light dark matter from neutrino experiments, Phys. Rev. D 100, 103011 (2019).

[62] D. Hooper and J. H. Steffen, Dark matter and the habitability of planets, J. Cosmol. Astropart. Phys. 07 (2012) 046.

[63] J. A. Caballero, A review on substellar objects beyond the deuterium burning mass limit: Planets, brown dwarfs or what?, arXiv:1808.07798.

[64] A. Gould et al. (MICROFUN, OGLE, ROBONET Collaborations), Microlens OGLE-2005-BLG-169 implies cool neptune-like planets are common, Astrophys. J. Lett. 644, L37 (2006).

[65] D. Saumon and M. S. Marley, The evolution of 1 and $t$ dwarfs in color-magnitude diagrams, Astrophys. J. 689, 1327 (2008).

[66] S. K. Leggett, P. Tremblin, T. L. Esplin, K. L. Luhman, and C. V. Morley, The y-type brown dwarfs: Estimates of mass and age from new astrometry, homogenized photometry, and near-infrared spectroscopy, Astrophys. J. 842, 118 (2017).

[67] C. Reylé, P. Delorme, C. J. Willott, L. Albert, X. Delfosse, T. Forveille, E. Artigau, L. Malo, G. J. Hill, and R. Doyon, The ultracool-field dwarf luminosity-function and space density from the canada-france brown dwarf survey, Astron. Astrophys. 522, A112 (2010).

[68] E. Buenzli, D. Apai, C. V. Morley, D. Flateau, A. P. Showman, A. Burrows, M. S. Marley, N. K. Lewis, and I. N. Reid, Vertical atmospheric structure in a variable brown dwarf: Pressure-dependent phase shifts in simultaneous hubble space telescope-spitzer light curves, Astrophys. J. 760, L31 (2012).

[69] B. A. Biller, I. J. M. Crossfield, L. Mancini, S. Ciceri, J. Southworth, T. G. Kopytova, M. Bonnefoy, N. R. Deacon, J. E. Schlieder, E. Buenzli et al., Weather on the nearest brown dwarfs: Resolved simultaneous multi-wavelength variability monitoring of wise J104915.57-531906.1AB, Astrophys. J. 778, L10 (2013).

[70] A. R. Zentner and A. P. Hearin, Asymmetric dark matter may alter the evolution of low-mass stars and brown dwarfs, Phys. Rev. D 84, 101302(R) (2011).

[71] A. Bouquet and P. Salati, Dark matter and the suppression of stellar core convection, Astrophys. J. 346, 284 (1989).

[72] P. Salati and J. Silk, A stellar probe of dark matter annihilation in galactic nuclei, Astrophys. J. 338, 24 (1989).

[73] I. V. Moskalenko and L. L. Wai, Dark matter burners, Astrophys. J. 659, L29 (2007).

[74] M. Fairbairn, P. Scott, and J. Edsjo, The zero age main sequence of WIMP burners, Phys. Rev. D 77, 047301 (2008). 
[75] D. Spolyar, K. Freese, and P. Gondolo, Dark Matter and the First Stars: A New Phase of Stellar Evolution, Phys. Rev. Lett. 100, 051101 (2008).

[76] K. Freese, P. Bodenheimer, D. Spolyar, and P. Gondolo, Stellar structure of dark stars: A first phase of stellar evolution resulting from dark matter annihilation, Astrophys. J. 685, L101 (2008).

[77] P. Scott, M. Fairbairn, and J. Edsjo, Dark stars at the Galactic centre-the main sequence, Mon. Not. R. Astron. Soc. 394, 82 (2009).

[78] M. Taoso, G. Bertone, G. Meynet, and S. Ekström, Dark matter annihilations in population III stars, Phys. Rev. D 78, 123510 (2008).

[79] K. Freese, P. Gondolo, J. A. Sellwood, and D. Spolyar, Dark matter densities during the formation of the first stars and in dark stars, Astrophys. J. 693, 1563 (2009).

[80] K. Freese, D. Spolyar, and A. Aguirre, Dark matter capture in the first star: A power source and a limit on stellar mass, J. Cosmol. Astropart. Phys. 11 (2008) 014.

[81] F. Iocco, Dark matter capture and annihilation on the first stars: Preliminary estimates, Astrophys. J. Lett. 677, L1 (2008).

[82] K. Freese, C. Ilie, D. Spolyar, M. Valluri, and P. Bodenheimer, Supermassive dark stars: Detectable in JWST, Astrophys. J. 716, 1397 (2010).

[83] D. Hooper, D. Spolyar, A. Vallinotto, and N. Y. Gnedin, Inelastic dark matter as an efficient fuel for compact stars, Phys. Rev. D 81, 103531 (2010).

[84] J. Casanellas and I. Lopes, Towards the use of asteroseismology to investigate the nature of dark matter, Mon. Not. R. Astron. Soc. 410, 535 (2011).

[85] E. Zackrisson, P. Scott, C.-E. Rydberg, F. Iocco, B. Edvardsson, G. Östlin, S. Sivertsson, A. Zitrin, T. Broadhurst, and P. Gondolo, Finding high-redshift dark stars with the james webb space telescope, Astrophys. J. 717, 257 (2010).

[86] F. Iocco, M. Taoso, F. Leclercq, and G. Meynet, Main Sequence Stars with Asymmetric Dark Matter, Phys. Rev. Lett. 108, 061301 (2012).

[87] C. Ilie, K. Freese, M. Valluri, I. T. Iliev, and P. R. Shapiro, Observing supermassive dark stars with james webb space telescope, Mon. Not. R. Astron. Soc. 422, 2164 (2012).

[88] I. Lopes and J. Silk, A particle dark matter footprint on the first generation of stars, Astrophys. J. 786, 25 (2014).

[89] J. Lopes, I. Lopes, and J. Silk, Asteroseismology of red clump stars as a probe of the dark matter content of the galaxy central region, Astrophys. J. 880, L25 (2019).

[90] E. Hassani, R. Pazhouhesh, and H. Ebadi, The effect of dark matter on stars at the galactic center: The paradox of youth problem, Int. J. Mod. Phys. D 29, 2050052 (2020).

[91] P. Mróz, A. Udalski, J. Skowron, R. Poleski, S. Kozłowski, M. K. Szymański, I. Soszyński, Ł. Wyrzykowski, P. Pietrukowicz, K. Ulaczyk et al., No large population of unbound or wide-orbit jupiter-mass planets, Nature (London) 548, 183 (2017).

[92] A. van Elteren, S. Portegies Zwart, I. Pelupessy, M. X. Cai, and S. L. W. McMillan, Survivability of planetary systems in young and dense star clusters, Astron. Astrophys. 624, A120 (2019).
[93] M. Perryman, J. Hartman, G. Á. Bakos, and L. Lindegren, Astrometric exoplanet detection with Gaia, Astrophys. J. 797, 14 (2014).

[94] G. F. Benedict, B. E. McArthur, G. Gatewood, E. Nelan, W. D. Cochran, A. Hatzes, M. Endl, R. Wittenmyer, S. L. Baliunas, G. A. H. Walker et al., The extrasolar planet epsilon eridani b: Orbit and mass, Astron. J. 132, 2206 (2006).

[95] F. Feng, M. Tuomi, and Hugh R. A. Jones, Detection of the closest Jovian exoplanet in the Epsilon Indi triple system, arXiv:1803.08163.

[96] J. Bailey, R. P. Butler, C. G. Tinney, H. R. A. Jones, S. O'Toole, B. D. Carter, and G. W. Marcy, A jupiter-like planet orbiting the nearby M dwarf GJ 832, Astrophys. J. 690, 743 (2009).

[97] R. P. Butler, J. A. Johnson, G. W. Marcy, J. T. Wright, S. S. Vogt, and D. A. Fischer, A long-period jupiter-mass planet orbiting the nearby M dwarf GJ 8491, Publ. Astron. Soc. Pac. 118, 1685 (2006).

[98] A. P. Hatzes, W. D. Cochran, M. Endl, E. W. Guenther, S. H. Saar, G. A. H. Walker, S. Yang, M. Hartmann, M. Esposito, D. B. Paulson et al., Confirmation of the planet hypothesis for the long-period radial velocity variations of beta geminorum, Astron. Astrophys. 457, 335 (2006).

[99] G. W. Marcy, R. P. Butler, D. A. Fischer, G. Laughlin, S. S. Vogt, G. W. Henry, and D. Pourbaix, A planet at 5 AU around 55 Cancri, Astrophys. J. 581, 1375 (2002).

[100] M. Mayor, S. Udry, D. Naef, F. Pepe, D. Queloz, N. C. Santos, and M. Burnet, The coralie survey for southern extra-solar planets, Astron. Astrophys. 415, 391 (2004).

[101] A. P. Hatzes, W. D. Cochran, M. Endl, B. McArthur, D. B. Paulson, G. A. H. Walker, B. Campbell, and S. Yang, A planetary companion to gamma Cephei A, Astrophys. J. 599, 1383 (2003).

[102] R. P. Butler, G. W. Marcy, D. A. Fischer, T. M. Brown, A. R. Contos, S. G. Korzennik, P. Nisenson, and R. W. Noyes, Evidence for multiple companions to $v$ andromedae, Astrophys. J. 526, 916 (1999).

[103] P. C. Gregory and D. A. Fischer, A bayesian periodogram finds evidence for three planets in 47 ursae majoris, Mon. Not. R. Astron. Soc. 403, 731 (2010).

[104] D. Naef, M. Mayor, S. G. Korzennik, D. Queloz, S. Udry, P. Nisenson, R. W. Noyes, T. M. Brown, J. L. Beuzit, C. Perrier et al., The elodie survey for northern extra-solar planets, Astron. Astrophys. 410, 1051 (2003).

[105] J. A. Johnson, R. P. Butler, G. W. Marcy, D. A. Fischer, S. S. Vogt, J. T. Wright, and K. M. G. Peek, A new planet around an $\mathrm{m}$ dwarf: Revealing a correlation between exoplanets and stellar mass, Astrophys. J. 670, 833 (2007).

[106] D. Fischer, P. Driscoll, H. Isaacson, M. Giguere, G. W. Marcy, J. Valenti, J. T. Wright, G. W. Henry, J. A. Johnson, A. Howard et al., Five planets and an independent confirmation of HD 196885AB from lick observatory, Astrophys. J. 703, 1545 (2009).

[107] R. Luque, T. Trifonov, S. Reffert, A. Quirrenbach, M. H. Lee, S. Albrecht, M. Fredslund Andersen, V. Antoci, F. Grundahl, C. Schwab et al., Precise radial velocities of giant stars, Astron. Astrophys. 631, A136 (2019).

[108] M. Endl, E. J. Brugamyer, W. D. Cochran, P. J. MacQueen, P. Robertson, S. Meschiari, I. Ramirez, M. Shetrone, 
K. Gullikson, M. C. Johnson et al., Two new long-period giant planets from the mcdonald observatory planet search and two stars with long-period radial velocity signals related to stellar activity cycles, Astrophys. J. 818, 34 (2016).

[109] B. D. Carter, R. P. Butler, C. G. Tinney, H. R. A. Jones, G. W. Marcy, C. McCarthy, D. A. Fischer, and A. J. Penny, A planet in a circular orbit with a 6 year period, Astrophys. J. 593, L43 (2003).

[110] J. Rey, G. Hébrard, F. Bouchy, V. Bourrier, I. Boisse, N. C. Santos, L. Arnold, N. Astudillo-Defru, X. Bonfils, S. Borgniet et al., The sophie search for northern extrasolar planets, Astron. Astrophys. 601, A9 (2017).

[111] G. W. Marcy, R. P. Butler, S. S. Vogt, D. A. Fischer, G. W. Henry, G. Laughlin, J. T. Wright, and J. A. Johnson, Five new extrasolar planets, Astrophys. J. 619, 570 (2005).

[112] S. S. Vogt, R. P. Butler, G. W. Marcy, D. A. Fischer, D. Pourbaix, K. Apps, and G. Laughlin, Ten low-mass companions from the keck precision velocity survey, Astrophys. J. 568, 352 (2002).

[113] S. W. Yee, E. A. Petigura, B. J. Fulton, H. A. Knutson, K. Batygin, G. Á. Bakos, J. D. Hartman, L. A. Hirsch, A. W. Howard, H. Isaacson et al., HAT-P-11: Discovery of a second planet and a clue to understanding exoplanet obliquities, Astron. J. 155, 255 (2018).

[114] J. T. Wright, S. Upadhyay, G. W. Marcy, D. A. Fischer, E. B. Ford, and J. A. Johnson, Ten new and updated multiplanet systems and a survey of exoplanetary systems, Astrophys. J. 693, 1084 (2009).

[115] A. Niedzielski, G. Nowak, M. Adamów, and A. Wolszczan, Substellar-mass companions to the K-dwarf $\mathrm{BD}+144559$ and the K-giants HD 240210 and BD + 20 2457, Astrophys. J. 707, 768 (2009).

[116] M. Marmier, D. Ségransan, S. Udry, M. Mayor, F. Pepe, D. Queloz, C. Lovis, D. Naef, N. C. Santos, R. Alonso et al., The coralie survey for southern extrasolar planets, Astron. Astrophys. 551, A90 (2013).

[117] D. Naef, M. Mayor, G. L. Curto, F. Bouchy, C. Lovis, C. Moutou, W. Benz, F. Pepe, D. Queloz, N. C. Santos et al., The harps search for southern extrasolar planets, Astron. Astrophys. 523, A15 (2010).

[118] R. P. Butler, G. W. Marcy, S. S. Vogt, D. A. Fischer, G. W. Henry, G. Laughlin, and J. T. Wright, Seven new keck planets orbiting $\mathrm{g}$ and $\mathrm{k}$ dwarfs, Astrophys. J. 582, 455 (2003).

[119] B. Sato, M. Omiya, R. A. Wittenmyer, H. Harakawa, M. Nagasawa, H. Izumiura, E. Kambe, Y. Takeda, M. Yoshida, Y. Itoh et al., A double planetary system around the evolved intermediate-mass star HD 4732, Astrophys. J. 762, 9 (2013).

[120] R. A. Wittenmyer, J. Horner, C. G. Tinney, R. P. Butler, H. R. A. Jones, M. Tuomi, G. S. Salter, B. D. Carter, F. E. Koch, S. J. O'Toole et al., The anglo-australian planet search. XXIII. Two new jupiter analogs, Astrophys. J. 783, 103 (2014).

[121] J. A. Valenti, D. Fischer, G. W. Marcy, J. A. Johnson, G. W. Henry, J. T. Wright, A. W. Howard, M. Giguere, and H. Isaacson, Two exoplanets discovered at keck observatory, Astrophys. J. 702, 989 (2009).
[122] L. Mancini, J. Lillo-Box, J. Southworth, L. Borsato, D. Gandolfi, S. Ciceri, D. Barrado, R. Brahm, and Th. Henning, Kepler-539: A young extrasolar system with two giant planets on wide orbits and in gravitational interaction, Astron. Astrophys. 590, A112 (2016).

[123] K. C. Sahu, S. Casertano, H. E. Bond, J. Valenti, T. Ed Smith, D. Minniti, M. Zoccali, M. Livio, N. Panagia, N. Piskunov et al., Transiting extrasolar planetary candidates in the galactic bulge, Nature (London) 443, 534 (2006).

[124] E. Bachelet and M. Penny, WFIRST and EUCLID: Enabling the microlensing parallax measurement from space, Astrophys. J. 880, L32 (2019).

[125] P. Mróz, R. Poleski, C. Han, A. Udalski, A. Gould, M. K. Szymański, I. Soszyński, P. Pietrukowicz, S. Kozłowski, J. Skowron et al., A free-floating or wide-orbit planet in the microlensing event OGLE-2019-BLG-0551, Astron. J. 159, 262 (2020).

[126] W. Zhu et al., mass measurements of isolated objects from space-based microlensing, Astrophys. J. 825, 60 (2016).

[127] Y. Shvartzvald et al., Spitzer microlensing parallax for OGLE-2017-BLG-0896 reveals a counter-rotating lowmass brown dwarf, Astron. J. 157, 106 (2019).

[128] J. Green et al., Wide-field infrared survey telescope (wfirst) final report, arXiv:1208.4012.

[129] S. A. Johnson, M. Penny, B. S. Gaudi, E. Kerins, N. J. Rattenbury, A. C. Robin, S. C. Novati, and C. B. Henderson, Predictions of the nancy grace roman space telescope galactic exoplanet survey. II. Free-floating planet detection rates, Astron. J. 160, 123 (2020).

[130] J. Stauffer, G. Helou, R. A. Benjamin, M. Marengo, J. D. Kirkpatrick, P. Capak, M. Kasliwal, J. M. Bauer, D. Minniti, J. Bally, N. Lodieu, B. Bowler, Z. Zhang, S. J. Carey, S. Milam, and B. Holler, The science advantage of a redder filter for wfirst, arXiv:1806.00554.

[131] D. B. Tilman Spohn and T. V. Johnson, Encyclopedia of the Solar System, Vol. 5 (Elsevier, New York, 2014).

[132] S. Auddy, S. Basu, and S. R. Valluri, Analytic models of brown dwarfs and the substellar mass limit, Adv. Astron. 2016, 1 (2016).

[133] O. Y. Gnedin, D. Ceverino, N. Y. Gnedin, A. A. Klypin, A. V. Kravtsov, R. Levine, D. Nagai, and G. Yepes, Halo contraction effect in hydrodynamic simulations of galaxy formation, arXiv:1108.5736.

[134] T. Cohen, M. Lisanti, A. Pierce, and T. R. Slatyer, Wino dark matter under siege, J. Cosmol. Astropart. Phys. 10 (2013) 061.

[135] J. Diemand, M. Kuhlen, and P. Madau, Dark matter substructure and gamma-ray annihilation in the Milky Way halo, Astrophys. J. 657, 262 (2007).

[136] J. Diemand, M. Kuhlen, P. Madau, M. Zemp, B. Moore, D. Potter, and J. Stadel, Clumps and streams in the local dark matter distribution, Nature (London) 454, 735 (2008).

[137] J. Stadel, D. Potter, B. Moore, J. Diemand, P. Madau, M. Zemp, M. Kuhlen, and V. Quilis, Quantifying the heart of darkness with GHALO — a multibillion particle simulation of a galactic Halo, Mon. Not. R. Astron. Soc.: Lett. 398, L21 (2009).

[138] V. Springel, J. Wang, M. Vogelsberger, A. Ludlow, A. Jenkins, A. Helmi, J. F. Navarro, C. S. Frenk, and S. D. M. 
White, The aquarius project: The subhaloes of galactic haloes, Mon. Not. R. Astron. Soc. 391, 1685 (2008).

[139] R. B. Metcalf and P. Madau, Compound gravitational lensing as a probe of dark matter substructure within galaxy halos, Astrophys. J. 563, 9 (2001).

[140] M. dal Ponte et al., Increasing the census of L and T dwarfs in wide binary and multiple systems using dark energy survey DR1 and gaia DR2 data, Mon. Not. R. Astron. Soc. 499, 5302 (2020).

[141] E. Valenti, M. Zoccali, O. A. Gonzalez, D. Minniti, J. Alonso-Garcia, E. Marchetti, M. Hempel, A. Renzini, and M. Rejkuba, Stellar density profile and mass of the Milky Way bulge from VVV data, Astron. Astrophys. 587, L6 (2016).

[142] E. Moulin (H. E. S. S. Collaboration), The inner 300 parsecs of the Milky Way seen by H. E. S. S.: A pevatron in the galactic centre, EPJ Web Conf. 136, 03017 (2017).

[143] C. M. Dutra, B. X. Santiago, and E. Bica, Low-extinction windows in the inner galactic bulge, Astron. Astrophys. 381, 219 (2002).

[144] K. Griest and D. Seckel, Cosmic asymmetry, neutrinos and the sun, Nucl. Phys. B283, 681 (1987); Erratum, Nucl. Phys. B296, 1034 (1988).

[145] N. A. Dondi, F. Sannino, and J. Smirnov, Thermal history of composite dark matter, Phys. Rev. D 101, 103010 (2020).

[146] R. Agnese et al. (SuperCDMS Collaboration), Low-mass dark matter search with CDMSlite, Phys. Rev. D 97, 022002 (2018).

[147] J. I. Collar, Search for a nonrelativistic component in the spectrum of cosmic rays at Earth, Phys. Rev. D 98, 023005 (2018).

[148] A. H. Abdelhameed et al. (CRESST Collaboration), First results from the CRESST-III low-mass dark matter program, Phys. Rev. D 100, 102002 (2019).

[149] D. Hooper and S. D. McDermott, Robust constraints and novel gamma-ray signatures of dark matter that interacts strongly with nucleons, Phys. Rev. D 97, 115006 (2018).

[150] R. Essig, A. Manalaysay, J. Mardon, P. Sorensen, and T. Volansky, First Direct Detection Limits on Sub-GeV Dark Matter from XENON10, Phys. Rev. Lett. 109, 021301 (2012).

[151] R. Essig, T. Volansky, and T.-T. Yu, New constraints and prospects for sub-GeV dark matter scattering off electrons in XENON, Phys. Rev. D 96, 043017 (2017).

[152] J. Angle, E. Aprile, F. Arneodo, L. Baudis, A. Bernstein, A. I. Bolozdynya, L. C. C. Coelho, C. E. Dahl, L. DeViveiros, A. D. Ferella et al., Search for Light Dark Matter in XENON10 Data, Phys. Rev. Lett. 107, 051301 (2011).

[153] E. Aprile, J. Aalbers, F. Agostini, M. Alfonsi, F. D. Amaro, M. Anthony, F. Arneodo, P. Barrow, L. Baudis, B. Bauermeister et al., Low-mass dark matter search using ionization signals in XENON100, Phys. Rev. D 94, 092001 (2016).

[154] P. Agnes, I. F. M. Albuquerque, T. Alexander, A. K. Alton, G. R. Araujo, D. M. Asner, M. Ave, H. O. Back, B. Baldin, G. Batignani et al., Constraints on Sub-Gev Dark-MatterElectron Scattering from the Darkside-50 Experiment, Phys. Rev. Lett. 121, 111303 (2018).
[155] R. Agnese, T. Aralis, T. Aramaki, I. J. Arnquist, E. Azadbakht, W. Baker, S. Banik, D. Barker, D. A. Bauer, T. Binder et al., First Dark Matter Constraints from a Supercdms Single-Charge Sensitive Detector, Phys. Rev. Lett. 121, 051301 (2018).

[156] M. Crisler, R. Essig, J. Estrada, G. Fernandez, J. Tiffenberg, M.S. Haro, T. Volansky, and T.-T. Yu, SENSEI: First Direct-Detection Constraints on Sub-GeV Dark Matter from a Surface Run, Phys. Rev. Lett. 121, 061803 (2018).

[157] O. Abramoff, L. Barak, I. M. Bloch, L. Chaplinsky, M. Crisler, Dawa, A. Drlica-Wagner, R. Essig, J. Estrada, E. Etzion et al., SENSEI: Direct-Detection Constraints on Sub-GeV Dark Matter from a Shallow Underground Run using a Prototype Skipper CCD, Phys. Rev. Lett. 122, 161801 (2019).

[158] A. Aguilar-Arevalo, D. Amidei, D. Baxter, G. Cancelo, B. A. Cervantes Vergara, A. E. Chavarria, E. Darragh-Ford, J. R. T. de Mello Neto, J. C. D'Olivo, J. Estrada et al., Constraints on Light Dark Matter Particles Interacting with Electrons from DAMIC at SNOLAB, Phys. Rev. Lett. 123, 181802 (2019).

[159] L. Barak et al., SENSEI: Direct-Detection Results on Sub-GeV Dark Matter from a New Skipper-CCD, Phys. Rev. Lett. 125, 171802 (2020).

[160] EDELWEISS Collaboration, First Germanium-Based Constraints on Sub-MeV Dark Matter with the EDELWEISS Experiment, Phys. Rev. Lett. 125, 141301 (2020).

[161] D. W. Amaral et al. (SuperCDMS Collaboration), Constraints on low-mass, relic dark matter candidates from a surface-operated SuperCDMS single-charge sensitive detector, Phys. Rev. D 102, 091101 (2020).

[162] H. An, M. Pospelov, J. Pradler, and A. Ritz, Directly Detecting MeV-Scale Dark Matter via Solar Reflection, Phys. Rev. Lett. 120, 141801 (2018); Erratum, Phys. Rev. Lett. 121, 259903 (2018).

[163] T. Emken, C. Kouvaris, and N. G. Nielsen, The sun as a sub-GeV dark matter accelerator, Phys. Rev. D 97, 063007 (2018).

[164] P. J. Fox and E. Poppitz, Leptophilic dark matter, Phys. Rev. D 79, 083528 (2009).

[165] J. Kopp, V. Niro, T. Schwetz, and J. Zupan, DAMA/ LIBRA and leptonically interacting dark matter, Phys. Rev. D 80, 083502 (2009).

[166] J. Kopp, L. Michaels, and J. Smirnov, Loopy constraints on leptophilic dark matter and internal bremsstrahlung, J. Cosmol. Astropart. Phys. 04 (2014) 022.

[167] N. F. Bell, Y. Cai, R. K. Leane, and A. D. Medina, Leptophilic dark matter with $Z^{\prime}$ interactions, Phys. Rev. D 90, 035027 (2014).

[168] F. D'Eramo, B. J. Kavanagh, and P. Panci, Probing leptophilic dark sectors with hadronic processes, Phys. Lett. B 771, 339 (2017).

[169] G. D. Starkman, A. Gould, R. Esmailzadeh, and S. Dimopoulos, Opening the window on strongly interacting dark matter, Phys. Rev. D 41, 3594 (1990).

[170] E. O. Nadler, V. Gluscevic, K. K. Boddy, and R. H. Wechsler, Constraints on dark matter microphysics from the Milky Way satellite population, Astrophys. J. Lett. 878, 
L32 (2019); Erratum, Astrophys. J. Lett. 897, L46 (2020); Erratum, Astrophys. J. Lett. 897, L46 (2020).

[171] E. O. Nadler et al. (DES Collaboration), Milky Way Satellite Census. III. Constraints on Dark Matter Properties from Observations of Milky Way Satellite Galaxies, Phys. Rev. Lett. 126, 091101 (2021).

[172] B. Dutta, S. Ghosh, and J. Kumar, A sub-GeV dark matter model, Phys. Rev. D 100, 075028 (2019).

[173] R. K. Leane, T. R. Slatyer, J. F. Beacom, and K. C. Y. Ng, GeV-scale thermal WIMPs: Not even slightly ruled out, Phys. Rev. D 98, 023016 (2018).

[174] R. K. Leane, Indirect detection of dark matter in the galaxy, arXiv:2006.00513.

[175] M. Cirelli, N. Fornengo, B. J. Kavanagh, and E. Pinetti, Integral X-ray constraints on sub-GeV dark matter, Phys. Rev. D 103, 063022 (2021).

[176] S. Knapen, T. Lin, and K. M. Zurek, Light dark matter: Models and constraints, Phys. Rev. D 96, 115021 (2017).

[177] F. Kahlhoefer, K. Schmidt-Hoberg, T. Schwetz, and S. Vogl, Implications of unitarity and gauge invariance for simplified dark matter models, J. High Energy Phys. 02 (2016) 016.

[178] N. F. Bell, Y. Cai, and R. K. Leane, Dark forces in the sky: Signals from Z' and the dark Higgs, J. Cosmol. Astropart. Phys. 08 (2016) 001.

[179] N. F. Bell, Y. Cai, and R. K. Leane, Impact of mass generation for spin-1 mediator simplified models, J. Cosmol. Astropart. Phys. 01 (2017) 039.

[180] M. Duerr, F. Kahlhoefer, K. Schmidt-Hoberg, T. Schwetz, and S. Vogl, How to save the WIMP: Global analysis of a dark matter model with two s-channel mediators, J. High Energy Phys. 09 (2016) 042.

[181] N. F. Bell, Y. Cai, J. B. Dent, R. K. Leane, and T. J. Weiler, Enhancing dark matter annihilation rates with dark bremsstrahlung, Phys. Rev. D 96, 023011 (2017).

[182] Y. Cui and F. D'Eramo, Surprises from complete vector portal theories: New insights into the dark sector and its interplay with Higgs physics, Phys. Rev. D 96, 095006 (2017).

[183] J. Brande, T. Barclay, J. E. Schlieder, E. D. Lopez, and E. V. Quintana, The feasibility of directly imaging nearby cold Jovian planets with MIRI/JWST, Astron. J. 159, 18 (2019).
[184] A. H. G. Peter, Dark matter in the solar system III: The distribution function of WIMPs at the Earth from gravitational capture, Phys. Rev. D 79, 103533 (2009).

[185] A. H. G. Peter, Dark matter in the solar system I: The distribution function of WIMPs at the Earth from solar capture, Phys. Rev. D 79, 103531 (2009).

[186] C. M. Dutra, B. X. Santiago, E. L. D. Bica, and B. Barbuy, Extinction within $10^{\circ}$ of the galactic centre using 2MASS, Mon. Not. R. Astron. Soc. 338, 253 (2003).

[187] A. Mitridate and A. Podo, Bounds on dark matter decay from $21 \mathrm{~cm}$ line, J. Cosmol. Astropart. Phys. 05 (2018) 069.

[188] L. Vocadlo, J. Brodholt, D. Alfe, M. J. Gillan and G. D. Price, The $a b$ initio simulation of the Earth's core, Phil. Trans. R. Soc. A 360, 1795 (2002).

[189] R. N. Manchester, G. B. Hobbs, A. Teoh, and M. Hobbs, The Australia telescope national facility pulsar catalogue, Astron. J. 129, 1993 (2005).

[190] M. Camenzind, Compact Objects in Astrophysics: White Dwarfs, Neutron Stars and Black Holes (Springer-Verlag, Berlin Heidelberg, 2007).

[191] R. Garani and S. Palomares-Ruiz, Dark matter in the Sun: Scattering off electrons vs nucleons, J. Cosmol. Astropart. Phys. 05 (2017) 007.

[192] H.-N. Lin and X. Li, The dark matter profiles in the Milky Way, Mon. Not. R. Astron. Soc. 487, 5679 (2019).

[193] A. Burkert, The structure of dark matter halos in dwarf galaxies, Astrophys. J. 447, L25 (1995).

[194] M. Pato, F. Iocco, and G. Bertone, Dynamical constraints on the dark matter distribution in the Milky Way, J. Cosmol. Astropart. Phys. 12 (2015) 001.

[195] J. Bramante, A. Delgado, and A. Martin, Multiscatter stellar capture of dark matter, Phys. Rev. D 96, 063002 (2017).

[196] T. Guillot and D. Gautier, Giant planets, arXiv:0912.2019.

[197] E. Aprile et al. (XENON Collaboration), Search for Light Dark Matter Interactions Enhanced by the Migdal Effect or Bremsstrahlung in XENON1T, Phys. Rev. Lett. 123, 241803 (2019).

[198] Z. Z. Liu et al. (CDEX Collaboration), Constraints on Spin-Independent Nucleus Scattering with sub-GeV Weakly Interacting Massive Particle Dark Matter from the CDEX-1B Experiment at the China Jinping Underground Laboratory, Phys. Rev. Lett. 123, 161301 (2019). 